\title{
The Association between Serum Gamma- Glutamyltransferase within Normal Levels and Metabolic Syndrome in Office Workers: A 4-Year Follow-up Study

\section{Byung-Seong Suh*}

Department of Occupational Medicine, Kangbuk Samsung Hospital, Sungkyunkwan University School of Medicine, Seoul, Korea

Background: Serum gamma-glutamyltransferase (GGT) has been suggested as a predictor for development of the metabolic syndrome in non-Korean population, but studies in Korean population are scarce. Therefore, we examined the association between serum GGT levels and the incidence of the metabolic syndrome in Korean male office workers.

Methods: The study population consisted of 32,692 office workers who underwent health checkups in both 2005 and 2009. A total of 17,583 with elevated GGT levels, the presence of metabolic syndrome, medication history at baseline, and female office workers were excluded. Finally, 15,109 subjects were included in the final analysis. We measured serum GGT levels and individual metabolic components.

Results: As a quartile of serum GGT increased, 4-year follow-up incidence of the metabolic syndrome increased. After adjustment for age, alcohol drinking status and smoking status in 2005, logistic regression analysis showed that the odds ratios (95\% confidence interval) for incident metabolic syndrome in 2009 compared to the lowest quartile and upper quartiles were 1.00 (reference), 1.57 (1.24-2.00), 2.73 (2.17-3.43), 3.78 (3.02-4.74), and statistically significant $(\mathrm{P}<0.001)$, respectively.

Conclusion: These results showed that the higher serum GGT predicted the future development of metabolic syndrome. In Korean male office workers without the metabolic syndrome, the serum GGT levels despite normal levels were associated with an increased risk of incident metabolic syndrome.

Keywords: Gamma-Glutamyltransferase; Metabolic Syndrome; Incidence

\section{INTRODUCTION}

Received: November 17, 2011, Accepted: January 17, 2012

${ }^{*}$ Corresponding Author: Byung-Seong Suh

Tel: +82-2-2001-1345, Fax: +82-2-2001-2748

E-mail: byungseong.suh@samsung.com

Korean Journal of Family Medicine

Copyright (C) 2012 The Korean Academy of Family Medicine

(a) This is an open-access article distributed under the terms of the Creative Commons Attribution Non-Commercial License (http://creativecommons.org/licenses/by-nc/3.0) which permits unrestricted noncommercial use, distribution, and reproduction in any medium, provided the original work is properly cited.
The common clustering of abdominal obesity, hypertriglyceridemia, low high density lipoprotein cholesterol (HDL-C), high blood glucose level and hypertension is referred to as metabolic syndrome (MetS), which is closely related to an increased risk for type 2 diabetes and cardiovascular disease. The prevalence of MetS in Korean male population over 30 years of age increased from $29.6 \%$ in $1998,31.5 \%$ in 2001 , and $33.5 \%$ in 2005, whereas in Korean female population over 30 years of 
age decreased from $30.3 \%$ in $1998,33.0 \%$ in 2001 , and $26.5 \%$ in 2005. ${ }^{1)}$ This could indicate an increasing trend in the prevalence of MetS among male Korean population and might be related to westernized lifestyle of Korean population, especially in male office workers.

Serum gamma-glutamyltransferase (GGT) has been used widely as an index of alcohol intake or liver dysfunction, ${ }^{2)}$ and has been one of the item as a part of national health checkup program in Korea. Previous studies suggest that GGT is closely related to risk factors for cardiovascular disease, hypertension, type 2 diabetes, and MetS. ${ }^{3,4)}$ Recently a cross-sectional study among the Korean population reported an association between GGT, MetS components and insulin resistance. ${ }^{5)}$ Additionally, elevated GGT concentrations are related to older age, male, smoking and physical inactivity, hypertension, hypertriglyceridemia, and low HDL-C. ${ }^{6-8)}$

The role of GGT is maintaining adequate levels of intracellular glutathione and serum GGT has been proposed as a marker of oxidative stress. ${ }^{9)}$ Serum GGT may be strongly associated with obesity or fat accumulation of liver and contributing to the development of insulin resistance. ${ }^{10)}$ Recent study showed that high GGT was an independent and early predictor for chronic disorders as well as cardiovascular disease. ${ }^{11)}$

Even though there are some cross-sectional studies regarding the association between GGT and MetS, longitudinal studies which can show a temporal relationship are scarce among the Korean population. Therefore, we hypothesized that GGT is a predictor for future development of MetS. We examined the association between GGT within normal range and the development of MetS among MetS-free male Korean workers. After 4 years, we analyzed the cumulative incidence of MetS in 2009 and investigated on how elevated GGT influenced MetS.

\section{METHODS}

\section{Study Population}

The study population was comprised of male workers who underwent a health checkup program in both 2005 and 2009. A total of 76,984 and 98,423 individuals participated in the health checkup in 2005 and 2009, respectively. Among them, 32,692 individuals with both laboratory results and questionnaire were considered for this study. We selected only office male workers and excluded 9,374 non-office workers and 1,557 female workers. We further excluded 1,011 subjects with hepatitis, chronic liver disease, malignancy, and renal failure and 3,305 subjects with MetS or with current medication history for hypertension or diabetes mellitus. Finally, a total of 3,341 with abnormal GGT levels defined as GGT of > $63 \mathrm{IU} / \mathrm{L}$ were excluded and 15,109 subjects were included for analysis.

\section{Measurements}

Health checkup examinations included data on medical history, medication use, health-related behaviors, anthropometric and physical measurements, and blood biochemical measurements. Body weight was measured with light clothing and without shoes to the nearest $0.1 \mathrm{~kg}$ using a digital scale. Height was measured to the nearest $0.1 \mathrm{~cm}$. Body mass index (BMI) was calculated as weight in kilograms divided by height in meters squared. Trained

Table 1. Characteristics of the study subjects in 2005.

\begin{tabular}{lc}
\hline \multicolumn{1}{c}{ Variables } & Men $(\mathrm{n}=15,109)$ \\
\hline Age $(\mathrm{y})$ & $41.3 \pm 5.9$ \\
GGT $(\mathrm{IU} / \mathrm{L})$ & $28.1 \pm 13.2$ \\
BMI $\left(\mathrm{kg} / \mathrm{m}^{2}\right)$ & $23.8 \pm 2.6$ \\
TG $(\mathrm{mg} / \mathrm{dL})$ & $127.1 \pm 65.9$ \\
HDL-C $(\mathrm{mg} / \mathrm{dL})$ & $50.7 \pm 9.9$ \\
SBP $(\mathrm{mm} \mathrm{Hg})$ & $112.8 \pm 12.8$ \\
DBP $(\mathrm{mm}$ Hg) & $75.7 \pm 8.6$ \\
Glucose $(\mathrm{mg} / \mathrm{dL})$ & $95.1 \pm 11.1$ \\
Drinking status & \\
None & $2,691(17.8)$ \\
$\leq 1-2$ times/wk & $11,206(74.2)$ \\
$>2$ times/wk & $1,212(8.0)$ \\
Smoking status & \\
None & $5,331(35.3)$ \\
Ex-smoking & $3,809(25.2)$ \\
Smoking & $5,969(39.5)$ \\
\hline
\end{tabular}

Values are presented as mean \pm SD or number (\%). GGT: gamma glutamyltransferase, BMI: body mass index, TG: triglyceride, HDL-C: high density lipoprotein cholesterol, SBP: systolic blood pressure, DBP: diastolic blood pressure. 
nurses measured sitting blood pressure (BP) with a mercury sphygmomanometer.

Blood specimens were sampled from the antecubital vein after more than 12 hours of fasting. Serum levels of fasting glucose, triglycerides, HDL-C, and GGT were measured using Bayer Reagent Packs on an automated chemistry analyzer (Advia 1650 Autoanalyzer; Bayer Diagnostics, Leverkusen, Germany). Fasting glucose was measured using Hexokinse method (Hitachi 747 automatic analyzer, Hitachi, Japan). The clinical laboratory has been accredited and participates annually in inspections and surveys by the Korean Association of Quality Assurance for Clinical Laboratories.

\section{Definition of Metabolic Syndrome}

MetS was defined using the modified National Cholesterol Education Program Adult Treatment III $^{12)}$ as the presence of 3 or more among the following criteria: 1) abdominal obesity; ${ }^{13)} 2$ ) fasting blood glucose $110 \mathrm{mg} / \mathrm{dL}$; 3) triglycerides $150 \mathrm{mg} / \mathrm{dL}$; 4) $\mathrm{HDL}-\mathrm{C}<40 \mathrm{mg} / \mathrm{dL}$; and 5) BP $\geq 130 / 85 \mathrm{~mm} \mathrm{Hg}$. Triglycerides, $\mathrm{HDL}-\mathrm{C}$, glucose, and BP were determined at each visit. Because measurement of waist circumference was not available for all subjects, we substituted abdominal obesity with overall adiposity for BMI of $\geq 25 \mathrm{~kg} / \mathrm{m}^{2}$, which has been proposed as a cut-off for the diagnosis of obesity in Asian populations. ${ }^{13)}$ Current user of blood glucose lowering agents was considered as having diabetes mellitus and current user of BP lowering agents was considered as having hypertension.

\section{Statistical Analyses}

The statistical analysis of the data was carried out using PAWS ver. 18.0 (SPSS Inc., Chicago, IL, USA). Student t-test were used to compare the characteristics of participants, which are age, triglyceride, HDL-C, systolic blood pressure (SBP), diastolic blood pressure (DBP), fasting glucose, and baseline GGT quartiles. $\chi^{2}$-tests were used to compare alcohol drinking status and smoking status. We analyzed trend test according to the 4 groups of GGT quatiles. We analyzed the cumulative incidence of MetS in 2009 according to the 4 groups of GGT quartiles in 2005. We analyzed the adjusted odds ratios (and 95\% confidence intervals) for incident MetS comparing the highest three quartiles of the baseline (in 2005) serum GGT to the lowest quartile using logistic regression analyses. Age, alcohol drinking status, and smoking status were adjusted as possible confounders in the model. All reported P-values are two tailed, and those $<0.05$ were considered statistically significant.

\section{RESULTS}

\section{Clinical Characteristics of the Subjects}

The basic characteristics of the study subjects are presented in the 15,109 male office workers (Table 1 ). The average age of the

Table 2. Characteristics according to the quartiles of serum GGT in 2005.

\begin{tabular}{|c|c|c|c|c|c|}
\hline \multirow{2}{*}{ Variables } & 1st quartile & 2nd quartile & 3rd quartile & 4th quartile & \multirow{2}{*}{ P for trend } \\
\hline & $1-17(\mathrm{IU} / \mathrm{L})$ & $18-25(\mathrm{IU} / \mathrm{L})$ & 26-36 (IU/L) & $37-63(\mathrm{IU} / \mathrm{L})$ & \\
\hline Number & 3,648 & 4,124 & 3,673 & 3,664 & $<0.001$ \\
\hline Age (y) & $40.4 \pm 5.9$ & $41.0 \pm 5.8$ & $41.8 \pm 5.9$ & $42.0 \pm 5.7$ & $<0.001$ \\
\hline BMI $\left(\mathrm{kg} / \mathrm{m}^{2}\right)$ & $22.7 \pm 2.4$ & $23.4 \pm 2.4$ & $24.2 \pm 2.5$ & $24.8 \pm 2.5$ & $<0.001$ \\
\hline $\mathrm{TG}(\mathrm{mg} / \mathrm{dL})$ & $99.9 \pm 47.4$ & $118.7 \pm 59.2$ & $136.2 \pm 66.8$ & $154.6 \pm 75.0$ & $<0.001$ \\
\hline HDL-C (mg/dL) & $51.4 \pm 10.2$ & $50.9 \pm 10.1$ & $50.2 \pm 9.7$ & $50.2 \pm 9.5$ & $<0.001$ \\
\hline $\mathrm{SBP}(\mathrm{mm} \mathrm{Hg})$ & $110.5 \pm 12.8$ & $112.5 \pm 12.6$ & $113.7 \pm 12.8$ & $114.5 \pm 12.7$ & $<0.001$ \\
\hline DBP (mm Hg) & $73.8 \pm 8.4$ & $75.3 \pm 8.3$ & $76.5 \pm 8.5$ & $77.2 \pm 8.6$ & $<0.001$ \\
\hline Glucose (mg/dL) & $93.2 \pm 9.3$ & $94.7 \pm 11.4$ & $95.7 \pm 11.5$ & $96.7 \pm 11.7$ & $<0.001$ \\
\hline
\end{tabular}

Values are presented as mean \pm SD.

GGT: gamma glutamyltransferase, BMI: body mass index, TG: triglyceride, HDL-C: high density lipoprotein cholesterol, SBP: systolic blood pressure, DBP: diastolic blood pressure. 
subjects was 41.3 years. The average BMI and blood pressure were $23.8 \mathrm{~kg} / \mathrm{m}^{2}$ and $112.8 / 75.7 \mathrm{~mm} \mathrm{Hg}$, respectively. The percentage of alcohol drinking status, which was more than 2 times per week, presented $8.0 \%$ and current smoking status presented $39.5 \%$.

\section{Clinical Characteristics According to the Quartiles of Serum GGT}

The subjects were classified according to the quartiles of serum GGT ( $\leq 63 \mathrm{IU} / \mathrm{L}$ in 2005), and their MetS components in 2005 were compared (Table 2). The categories of the 4 quartiles of serum GGT were 1-17 IU/L, 18-25 IU/L, 26-36 IU/L, and 37-63 IU/L, respectively. The numbers of 4 quartiles of serum GGT were 3,648 (24.1\%), 4,124 (27.3\%), 3,673 (24.3\%), and 3,664 (24.3\%), respectively. The trend of age, BMI, GGT, triglyceride, SBP, and DBP increased from the first quartile to the fourth quartile $(\mathrm{P}<0.001)$. HDL-C decreased from the first quartile to the fourth quartile $(\mathrm{P}<0.001)$.

The subjects were classified according to the 4 quartiles of serum GGT ( $\leq 63 \mathrm{IU} / \mathrm{L}$ in 2005), and their MetS components in 2009 were compared (Table 3). The trend of age, BMI, GGT, triglyceride, SBP, and DBP increased from the first quartile to the fourth quartile $(\mathrm{P}<0.001)$. HDL-C decreased from the first quartile to the fourth quartile $(\mathrm{P}<0.001)$.

\section{Cumulative Incidence and Odds Ratios of MetS According to the 4 Quartiles of Serum GGT}

We defined incidence of MetS as the percentage of newly number in MetS in 2009/number of MetS-free and GGT in normal levels in 2005. Total percentage (number) of incidence of MetS were $7.4 \%(1,113)$. The respective percentage (number) of cumulative incidence were 3.4\% (123), 5.4\% (221), 8.8\% (325),

Table 3. Characteristics according to the quartiles of serum GGT in 2009.

\begin{tabular}{|c|c|c|c|c|c|}
\hline \multirow{2}{*}{ Variables } & 1st quartile & 2nd quartile & 3rd quartile & 4th quartile & \multirow{2}{*}{ P for trend } \\
\hline & $1-17$ (IU/L) & 18-25 (IU/L) & 26-36 (IU/L) & 37-63 (IU/L) & \\
\hline Number & 3,648 & 4,124 & 3,673 & 3,664 & $<0.001$ \\
\hline BMI $\left(\mathrm{kg} / \mathrm{m}^{2}\right)$ & $22.9 \pm 2.4$ & $23.6 \pm 2.4$ & $24.3 \pm 2.5$ & $24.9 \pm 2.6$ & $<0.001$ \\
\hline TG (mg/dL) & $111.2 \pm 59.7$ & $127.7 \pm 67.8$ & $144.2 \pm 73.5$ & $162.4 \pm 91.0$ & $<0.001$ \\
\hline HDL-C (mg/dL) & $53.5 \pm 11.2$ & $53.1 \pm 11.0$ & $51.8 \pm 10.7$ & $51.7 \pm 10.5$ & $<0.001$ \\
\hline SBP (mm Hg) & $113.6 \pm 10.8$ & $115.0 \pm 10.7$ & $116.0 \pm 10.9$ & $117.0 \pm 11.0$ & $<0.001$ \\
\hline DBP (mm Hg) & $73.8 \pm 7.9$ & $74.8 \pm 8.1$ & $75.7 \pm 8.2$ & $76.4 \pm 8.4$ & $<0.001$ \\
\hline Glucose (mg/dL) & $95.0 \pm 12.3$ & $96.5 \pm 12.1$ & $97.6 \pm 12.4$ & $99.1 \pm 13.9$ & $<0.001$ \\
\hline
\end{tabular}

Values are presented as mean $\pm \mathrm{SD}$.

GGT: gamma glutamyltransferase, BMI: body mass index, TG: triglyceride, HDL-C: high density lipoprotein cholesterol, SBP: systolic blood pressure, DBP: diastolic blood pressure.

Table 4. Cumulative incidence of metabolic syndrome according to the quartiles of serum GGT.

\begin{tabular}{|c|c|c|c|c|}
\hline \multirow{2}{*}{ Variables } & 1st quartile & 2nd quartile & 3rd quartile & 4th quartile \\
\hline & $1-17(\mathrm{IU} / \mathrm{L})$ & 18-25 (IU/L) & 26-36 (IU/L) & 37-63 (IU/L) \\
\hline Number & 3,648 & 4,124 & 3,673 & 3,664 \\
\hline \multicolumn{5}{|l|}{ Metabolic syndrome } \\
\hline Number & 123 & 221 & 325 & 444 \\
\hline Incidence* & 3.4 & 5.4 & 8.8 & 12.1 \\
\hline
\end{tabular}

GGT: gamma glutamyltransferase.

*Cumulative incidence (\%, incidence case of metabolic syndrome subjects among total subjects of each quartile during the follow-up). 
and $12.1 \%$ (444), as quartiles, respectively. As higher the GGT quartile, the percentage of incidence of MetS increased (Table 4).

To confirm that the higher GGT quartile increased incident MetS more than the lower GGT quartile, we adjusted age, alcohol drinking status and smoking status in 2005. We analyzed the 4 GGT quartiles according to MetS by logistic regression. The odds ratios (confidence interval) of MetS were 1.57 (1.24-2.00), 2.73 (2.17-3.43), and 3.78 (3.02-4.74), respectively, and statistically significant $(P<0.001)$. The positive association between the baseline GGT levels and incident MetS was observed $(\mathrm{P}<0.001)$. The odds ratio of alcohol drinking status was not statistically significant $(\mathrm{P}>0.05)$. The odds ratios of current and past smoking status were higher than non-smoking status (Table 5).

\section{DISCUSSION}

In numerous studies, the relationship between GGT and various diseases such as cardiovascular disease, hypertension and diabetes mellitus was proven to be relevant and henceforth, it was shown to be useful in predicting development of MetS. Unlike the other existing cross-sectional studies, this study investigated the factors that have an effect on the development of MetS by carrying out a longitudinal study (from 2005 to 2009) that looked into male subjects who completed medical examination showing MetS-free and a normal range of GGT; the normal range of GGT was divided into 4 quartiles, and the factors that influenced MetS were investigated 4 years later. As a result of this study, even though the subjects were within the normal range of GGT, the higher GGT quartiles were shown to have MetS similarly at a higher rate, than the ones in the lower quartiles after 4 years of the initial carrying out of the study. This suggests that there is a close relationship between an increase of GGT and MetS.

According to the results of the study after 4 years, the incidence rate of MetS in 2009 was $7.4 \%$, which is lower than the result of a study that investigated subjects without MetS after 3 years $^{14)}$ with $10.72 \%$ rate. However, the incidence rate of MetS could have been high as the study investigated 429 subjects, were relatively small number of subjects, and the average age was high with 48.2.

According to the results of each variable of MetS compared with the serum GGT divided into 4 quartiles, both in 2005

Table 5. Adjusted odds ratios and 95\% confidence interval in the quartiles of serum GGT level for the incidence of metabolic syndrome by logistic regression analysis.

\begin{tabular}{|c|c|c|c|}
\hline Variables* & Odds ratio & 95\% confidence interval & P-value \\
\hline \multicolumn{4}{|l|}{ GGT } \\
\hline 1st quartile & 1.00 & Reference & - \\
\hline 2nd quartile & 1.57 & $1.24-2.00$ & $<0.001$ \\
\hline 3rd quartile & 2.73 & $2.17-3.43$ & $<0.001$ \\
\hline 4th quartile & 3.78 & $3.02-4.74$ & $<0.001$ \\
\hline Age (y) & 1.03 & $1.02-1.04$ & $<0.001$ \\
\hline \multicolumn{4}{|l|}{ Drinking status in 2005} \\
\hline None & 1.00 & Reference & - \\
\hline$\leq 1-2$ times $/ w k$ & 0.89 & $0.75-1.05$ & 0.082 \\
\hline$>2$ times/wk & 0.74 & $0.57-0.96$ & 0.173 \\
\hline \multicolumn{4}{|l|}{ Smoking status in 2005} \\
\hline None & 1.00 & Reference & - \\
\hline Ex-smoker & 1.12 & $0.95-1.33$ & $<0.001$ \\
\hline Current smoker & 1.32 & $1.14-1.53$ & $<0.001$ \\
\hline
\end{tabular}

GGT: gamma glutamyltransferase. 
and 2009 results showed that the higher the quartile of serum GGT, age, triglyceride, SBP, DBP, and fasting glucose similarly increased which were statistically significant. The higher the quartile of serum GGT, only HDL-C was similarly decreased and statistically significant. MetS components were considerably under the influence of GGT.

According to the result of a research in Framingham Heart Study that tracked records of subjects without MetS for 19 years, ${ }^{15)}$ MetS increased in the case of a high GGT rate during the observation period. Furthermore, in the case of a research that observed subjects with a normal GGT for 3 years, ${ }^{14)}$ compared to the basal GGT 1 quartile, the study showed similar results with an increased rate of 2.28, 1.53, and 4.56 times, age 1.06 times and drinking status 1.35 times, respectively. However, in the present study alcohol has shown different results. There was a limitation to this research as the survey on alcohol drinking status merely asked a question on the frequency that a person consumes alcoholic drink per week. The similar results were not attainable for smoking status, as the information analyzed was categorized merely into non-smokers, past smokers and current smokers.

According to the data from an Epidemiological Study on the Insulin Resistance Syndrome (Canadian Intercollegiate Sport Injury Registry), ${ }^{16)}$ it showed similar results $1.96,2.25$, and 3.81 compared to the basal GGT 1 quartile, even after an adjustment of variables such as age, drinking, smoking, and exercise status. From this research, it is also evident that within a normal range group, the higher GGT, the more risks it has of getting MetS.

From this research, it is also evident that within a normal range group, the higher GGT rate, the more risks it has of getting MetS. This shows a close correlation between GGT and MetS, and it would mean even within a range group with a normal GGT without MetS, the higher GGT rate, the more risk it has of getting MetS hereafter.

Ruttmann et al. ${ }^{17)}$ carried out a cross-sectional and longitudinal study against 163,944 Australians and concluded that GGT is a risk factor for cardiovascular disease and in the highest GGT quartile, the number of people with vascular disease increased 64 percent for males, and 51 percent for females, respectively. In particular, it was confirmed that GGT is a key dependent variable for the death caused by cardiovascular failure due to chronic heart disease. Kazemi-Shirazi et al. ${ }^{18)}$ also carried out a longitudinal study against 283,438 participants for 12 years, and suggested that even within the normal group, the highest GGT quartile increases the cause of death by $100 \%$ and therefore, GGT is a strong factor for long-term survival.

There are not enough records that explain the correlation between MetS and GGT, but the first record shows that the high GGT in a normal scope is an expression of non-alcoholic fatty liver that is closely related to the deposit of visceral fat and obesity. ${ }^{11)}$ The second record states that the increase of GGT causes inflammatory reaction and such reaction hinders not only the metabolism of insulin of a liver but also of a number of other organs. ${ }^{19,20)}$ The third record argues that the increase of GGT results from an oxidative stress and it plays a key role in the revelation of diabetes mellitus and MetS. ${ }^{21)}$

This study is significant that unlike the other cross-sectional studies carried out with only a few numbers of subjects, it was a four-year retrospective cohort research with a large number of subjects. Unlike other studies, the observation of incidence is possible, so it should provide important information on the concentration of GGT and the development of MetS.

The limitation of this study is firstly that it is based on data from medical examinations of only one university hospital and a categorized group, office workers and they cannot represent the whole population. Secondly, the investigations of the factors that can influence GGT and the development of MetS such as the ability of taking drugs, diet habit, and alcohol drinking status and smoking status are inadequate. Thirdly, because serum GGT can increase in the case of subclinical liver disorder, such subjects could have been included in the study. Fourthly, observation period is only four years and needs a long-term observation. Hence, a more successful research will be achieved if a foothold is gained for a long-term large scale prospective research through a cooperative study between numerous institutions complementing the above-mentioned limitations.

To conclude, the study shows that even in a group without a presence of MetS and the basal GGT at a normal level, the basal GGT belongs to a higher group that has a greater risk of getting MetS. Furthermore, it implies that GGT is related to a risk factor of cardiovascular diseases that triggers MetS and an increase of serum GGT can be henceforth a useful factor in predicting the development of MetS. 


\section{CONFLICT OF INTEREST}

No potential conflict of interest relevant to this article was reported.

\section{REFERENCES}

1. The Ministry of Health and Welfare. The Third Korea National Health \& Nutrition Examination Survey (KNHANES III), 2005. Seoul: The Ministry of Health and Welfare; 2006.

2. Jousilahti P, Rastenyte D, Tuomilehto J. Serum gammaglutamyl transferase, self-reported alcohol drinking, and the risk of stroke. Stroke 2000;31:1851-5.

3. Wannamethee G, Ebrahim S, Shaper AG. Gammaglutamyltransferase: determinants and association with mortality from ischemic heart disease and all causes. Am J Epidemiol 1995;142:699-708.

4. Kim DJ, Noh JH, Cho NH, Lee BW, Choi YH, Jung JH, et al. Serum gamma-glutamyltransferase within its normal concentration range is related to the presence of diabetes and cardiovascular risk factors. Diabet Med 2005;22:1134-40.

5. Kang YH, Min HK, Son SM, Kim IJ, Kim YK. The association of serum gamma glutamyltransferase with components of the metabolic syndrome in the Korean adults. Diabetes Res Clin Pract 2007;77:306-13.

6. Nakanishi N, Suzuki K, Tatara K. Serum gammaglutamyltransferase and risk of metabolic syndrome and type 2 diabetes in middle-aged Japanese men. Diabetes Care 2004;27:1427-32.

7. Rantala AO, Lilja M, Kauma H, Savolainen MJ, Reunanen A, Kesaniemi YA. Gamma-glutamyl transpeptidase and the metabolic syndrome. J Intern Med 2000;248:230-8.

8. Pintus F, Mascia P. Distribution and population determinants of gamma-glutamyltransferase in a random sample of Sardinian inhabitants. 'ATS-SARDEGNA' Research Group. Eur J Epidemiol 1996;12:71-6.

9. Lee DH, Blomhoff R, Jacobs DR Jr. Is serum gamma glutamyltransferase a marker of oxidative stress? Free Radic Res 2004;38:535-9.
10. Marchesini G, Brizi M, Bianchi G, Tomassetti S, Bugianesi E, Lenzi M, et al. Nonalcoholic fatty liver disease: a feature of the metabolic syndrome. Diabetes 2001;50:1844-50.

11. Lee DH, Jacobs DR Jr, Gross M, Kiefe CI, Roseman J, Lewis CE, et al. Gamma-glutamyltransferase is a predictor of incident diabetes and hypertension: the Coronary Artery Risk Development in Young Adults (CARDIA) Study. Clin Chem 2003;49:1358-66.

12. Expert Panel on Detection, Evaluation, and Treatment of High Blood Cholesterol in Adults. Executive Summary of The Third Report of The National Cholesterol Education Program (NCEP) Expert Panel on Detection, Evaluation, And Treatment of High Blood Cholesterol In Adults (Adult Treatment Panel III).JAMA 2001;285:2486-97.

13. World Health Organization Western Pacific Region; International Association for the Study of Obesity; International Obesity Task Force. The Asia-Pacific perspective: redefining obesity and its treatment. Sydney: Health Communications Australia Pty Ltd.; 2000.

14. Oh SW, Lee JG, Kim MS, Jung DW, Kim YJ, Lee S, et al. Relations of serum gamma-glutamyltransferase levels to incidence of the metabolic syndrome. J Korean Acad Fam Med 2008;29:838-43.

15. Lee DS, Evans JC, Robins SJ, Wilson PW, Albano I, Fox CS, et al. Gamma glutamyl transferase and metabolic syndrome, cardiovascular disease, and mortality risk: the Framingham Heart Study. Arterioscler Thromb Vasc Biol 2007;27:127-33.

16. Andre P, Balkau B, Vol S, Charles MA, Eschwege E; DESIR Study Group. Gamma-glutamyltransferase activity and development of the metabolic syndrome (International Diabetes Federation Definition) in middle-aged men and women: Data from the Epidemiological Study on the Insulin Resistance Syndrome (DESIR) cohort. Diabetes Care 2007;30:2355-61.

17. Ruttmann E, Brant LJ, Concin H, Diem G, Rapp K, Ulmer H, et al. Gamma-glutamyltransferase as a risk factor for cardiovascular disease mortality: an epidemiological investigation in a cohort of 163,944 Austrian adults. Circulation 2005;112:2130-7.

18. Kazemi-Shirazi L, Endler G, Winkler S, Schickbauer T, Wagner O, Marsik C. Gamma glutamyltransferase and longterm survival: is it just the liver? Clin Chem 2007;53:940-6. 
19. Pradhan AD, Manson JE, Rifai N, Buring JE, Ridker PM. C-reactive protein, interleukin 6, and risk of developing type 2 diabetes mellitus. JAMA 2001;286:327-34.

20. Freeman DJ, Norrie J, Caslake MJ, Gaw A, Ford I, Lowe $\mathrm{GD}$, et al. C-reactive protein is an independent predictor of risk for the development of diabetes in the West of Scotland
Coronary Prevention Study. Diabetes 2002;51:1596-600.

21. Ceriello A, Motz E. Is oxidative stress the pathogenic mechanism underlying insulin resistance, diabetes, and cardiovascular disease? The common soil hypothesis revisited. Arterioscler Thromb Vasc Biol 2004;24:816-23. 